\title{
Anabases
}

ANABASES Traditions et réceptions de l'Antiquité

$18 \mid 2013$

Varia

\section{Lawrence Durrell, les ruines et l'histoire}

Isabelle Tassignon

\section{OpenEdition}

Journals

Édition électronique

URL : http://journals.openedition.org/anabases/4462

DOI : 10.4000/anabases.4462

ISSN : 2256-9421

\section{Éditeur}

E.R.A.S.M.E.

\section{Édition imprimée}

Date de publication : 1 octobre 2013

Pagination : 197-212

ISSN : 1774-4296

\section{Référence électronique}

Isabelle Tassignon, «Lawrence Durrell, les ruines et l'histoire », Anabases [En ligne], 18 | 2013, mis en ligne le 01 novembre 2016, consulté le 20 octobre 2019. URL : http://journals.openedition.org/ anabases/4462 : DOI : 10.4000/anabases.4462 


\section{Lawrence Durrell, les ruines et l'histoire}

L'ÉCRIVAIn BRITANNIQUe Lawrence Durrell aurait eu cent ans le 12 février 2012, l'occasion pour l'International Lawrence Durrell Society d'organiser un beau congrès pour fêter l'événement ${ }^{1}$. L'auteur du Quatuor d'Alexandrie et du Quintet d'Avignon avait fait de la Méditerranée sa terre d'élection, en s’installant avec toute sa famille, dès 1935, à Corfou ; pourtant, rien ne prédisposait ce jeune homme né aux Indes à faire de la Grèce son paradis, si ce n'est sa lumière et son climat ${ }^{2}$; il occupe ensuite divers postes diplomatiques au service de la Couronne en Égypte, à Rhodes, à Chypre (avec deux inter-

1 Londres, Goodenough College et British Library, 13-18 juin 2012, http://www.lawrencedurrell.org. Le français a toujours été pour L. Durrell une importante langue de communication; il débute en français en 1935 (L. Durrell, H. Miller, Correspondance 1935-1980 [éd. I. S. MacNiven], Londres et New York, 1988, trad. française B. Willerval, F.J. Temple, F. Jaouën, Paris, Buchet/Chastel, 2004, p. 30) ; résidant à Sommières à partir de 1957, il s'exprime en français et contrôle lui-même la qualité des traductions françaises de ses œuvres. Il a également accordé des interviews en français (notamment à M. Alyn dans Le grand suppositoire, entretiens avec Marc Alyn, 1972, rééd. 2007, ou à Jacques Chancel, dans l'émission radiophonique Radioscopie du 17 février 1982). C'est la raison pour laquelle nous ferons ici référence aux traductions françaises de l'œuvre de L. Durrell.

2 Lire à ce propos les lignes que G. Durrell consacre à la décision du départ pour la Grèce dans My Family and other Animals, Londres, 1956 ; trad. française L. LACK, Ma famille et autres animaux, Paris, 1958 ; édition utilisée ici : Paris, Gallmeister, 2007, p. 18-19 ("J'ai reçu ce matin une lettre de George [Wilkinson]... il dit que Corfou est merveilleux. Pourquoi ne pas partir en Grèce ?") ; voir aussi L. DurrelL, L'esprit des lieux. Lettres et essais de voyage, trad. française J.-R. MajOR, Paris, Gallimard, 1976 (1969), p. 24. 
mèdes en Argentine et en Yougoslavie). Après son installation à Sommières (Gard), il retourne souvent en Grèce, pour " retrouver comme un archéologue les fantômes qui hantent des pays qui ont tant changé ${ }^{3}$ ». Pour notre part, le propos sera d'aborder les liens entre l'écrivain, l'archéologie et l'histoire dans ses récits de voyage 4 et dans sa correspondance $^{5}$ et, en tentant de cerner ses principales sources, d'apprécier la qualité de son information en ces deux domaines. On s'interrogera ensuite sur le sort à réserver aux informations archéologiques ou historiques qu'il est le seul à donner, pour répondre à la question suivante : dans quelle mesure les informations livrées par L. Durrell à son lecteur, qu'il soit voyageur, historien ou archéologue, sont-elles fiables?

Si les vestiges archéologiques ne constituent évidemment pas le propos principal de L. Durrell, l'Antiquité, essentiellement grecque, apparaît en filigrane de la plupart de ses ouvrages, de L'île de Prospéro (sur ses années de jeunesse à Corfou, publié en 1945) aux Îles grecques (1978). Ni les artefacts, ni les ruines, les sites fouillés ou les restaurations ne le laissent indifférent et avoir participé, en tant que directeur des presses du gouvernement à Rhodes, à la publication puis à la diffusion de Clara Rodos, l'ouvrage en neuf volumes consacré aux fouilles italiennes à Rhodes, n'était pas la moindre de ses fiertés ${ }^{6}$. À vrai dire, l'archéologie qu'il découvre à son arrivée en Grèce au début des années 30 tourne au ralenti ; en effet, après les fouilles spectaculaires et prestigieuses de l'extrême fin du XIX ${ }^{\mathrm{e}}$ siècle et celles du début du XX ${ }^{\mathrm{e}}$ siècle (Mycènes, Delphes, Cnossos, Pergame...) et la restauration des ruines, la diminution des ressources financières des pays européens impliqués dans la Grande Guerre a entraîné une nette baisse des $\operatorname{travaux}^{7}$; dans la Grèce occupée, les fouilles sont à l'arrêt (sauf pour quelques équipes des forces d'occupation) et les équipes d'archéologues travaillent sur le matériel mis au jour antérieurement ${ }^{8}$. Après la Seconde Guerre mondiale, les recherches restent dans la continuité de ce qui avait été entrepris avant-guerre ${ }^{9}$. Les sites dont parle L. Durrell et

3 C. Alexandre-Garner, Lawrence Durrell. Dans l'ombre du soleil grec, Paris, 2011, p. 245, n. 10 : propos tenus par L. Durrell à propos de deux films de la BBC Television, 1976-1977, "Lawrence Durrell's Egypt » et «Lawrence Durrell's Greece », pour lesquels l'auteur était retourné en Égypte et en Grèce.

4 Cette enquête portera donc sur L'̂̀le de Prospéro, Londres, 1945, trad. française R. Giroux, Paris, Buchet/Chastel, 1962 ; Vénus et la mer, Londres, 1953, trad. française R. Giroux, Paris, Buchet/Chastel, 1969 ; Citrons acides, Londres, 1957, trad. française R. Giroux, Paris, Buchet/Chastel, 1961 ; Le Carrousel sicilien, Londres, 1977, trad. française P. GuIvarch, Paris, Gallimard, 1979 ; Les îles grecques, Londres 1978, trad. française D. Coste, Paris, Albin Michel, 1978 ; édition utilisée ici : Paris, Bartillat, 2010.

5 Publiée dans L'esprit des lieux (voir supra, n. 2) et dans Correspondance (voir supra, n. 1).

6 Les îles grecques, p. 142.

7 A. Bielman, dans Deux archéologues suisses photographient la Grèce, W. Deonna et P. Collart 1904-1939, Athènes-Genève, 2001, p. 19.

8 I. Morris, "Archaeologies of Greece ", dans ID., Classical Greece : Ancient Histories and Modern Archaeologies, Cambridge, 1994, p. 8-47, et particulièrement p. 35. 
qu'il fera découvrir à ses amis - et notamment à Henry Miller - ont été fouillés voire reconstruits quelques trente ans plus tôt. Rhodes et Cos se démarquent de ce paysage puisque, sous l'occupation italienne (1912-1947), plusieurs de leurs sites archéologiques sont fouillés, restaurés et, dans une large mesure, "remontés " (les remparts de la vieille ville de Rhodes, le temple d'Athéna à Lindos, l'Asclépieion de Cos...).

\section{L'archéologie ou le monde des incertitudes}

L'Italie n'apparaît dans l'œuvre durrellienne que par contraste avec la Grèce ; néanmoins, ses vestiges romains lui paraissent directement lisibles et compréhensibles. Des sites archéologiques de Grèce, il ne retire qu'une impression de grande confusion ${ }^{10}$; mais c'est avec le monde grec qu'il se sent pourtant des affinités électives.

Il a vu beaucoup de sites, tant en Grèce continentale et insulaire qu'à Chypre, mais essentiellement ce qui était visible là où il était ou ce qui était accessible en auto ; car, ainsi que le relève dans un ouvrage à visées touristiques l'archéologue F. Chapouthier ${ }^{11}$, l'automobile a contribué à élargir considérablement les horizons, y compris ceux des archéologues. Un grand absent toutefois : le temple d'Apollon Epikourios à Bassae, le site le mieux conservé de Grèce, mais relativement à l'écart des routes. À cette exception près, il a vu presque tous les sites classiques. Il a donc eu sous les yeux ce qu'il y avait de plus directement compréhensible parmi les sites archéologiques. Cependant, la préhistoire l'intéresse peu : il ne dit rien ou quasi rien des sites importants de l'archéologie cycladique - son commentaire sur l'île de Syros, si importante dans le monde de l'archéologie préhistorique pour ses "frying pans ", en dit long sur son peu d'intérêt pour cette période ${ }^{12}$. Par contre, lorsque la préhistoire se charge de merveilleux, de féerie ou de magie, il ne rechigne pas à en parler : ainsi évoque-t-il dans trois livres différents trois îles ayant livré des restes préhistoriques d'éléphants et d'hippopotames nains (Chypre, la Crète et la Sicile) ${ }^{13}$ ou le disque de Phaïstos, qui aurait " des pouvoirs magiques ${ }^{14}$ ".

10 L'esprit des lieux, p. 315 ("Delphes ») : «Là [en Grèce], tout est différent de l'Italie où la beauté provient de la civilisation - l'empreinte de la main humaine et sage est sur toutes choses. Tout y a une histoire, peut être remonté à ses origines, décodé, compris. Mais en Grèce... tout est confus, mêlé, déformé, brûlé par le soleil, explosé ".

11 A. Bon, F. Chapouthier, En Grèce, Paris, 1932, p. 7.

12 Les îles grecques, p. 296 : "À terre, il y a peu à voir et encore moins à apprendre sur le plan historique".

13 À Chypre, près de Kyrenia : Citrons acides, p. 135 ; en Crète : Les îles grecques, p. 70, citant un auteur qu'il ne nomme pas : "On nous dit qu'on a trouvé des os d'éléphants et d'hippopotames nains dans des sédiments troglodytiques géologiquement récents et que le cerf n'a disparu qu'à l'époque historique »; Carrousel sicilien, p. 123.

Les îles grecques, p. 101. 
Il conserve une vision romantique des ruines : elles constituent en quelque sorte la ponctuation du paysage grec, d'un pathétique sauvage ${ }^{15}$, accréditant par leur présence l'authenticité d'un site ; une cité non totalement dégagée - comme Camiros - est d'une "beauté chantante ${ }^{16}$ ". L'absence de ruines en un lieu restauré - comme l'acropole de Lindos - laisse planer une impression d'irréalité ${ }^{17}$; mais trop nombreuses, elles sont ennuyeuses ${ }^{18}$. De ce point de vue, il se démarque finalement peu de son cercle d'amis voyageurs. L'amie Freya Stark, par exemple, déclare "qu'elle préfere les amas de ruines encore non touchés par l'archéologue ${ }^{19}$ ". Mais d'autre part, L. Durrell est un homme qui aime les savoirs structurés, chronologiquement établis : d'où la présence discrète, mais récurrente dans son œuvre (de L'̂̀le de Prospéro aux Îles grecques, en passant par Vénus et la mer) d'éléments de chronologies, absolues ou relatives, voire des tableaux chronologiques ${ }^{20}$. Et en fait de ruines, L. Durrell voudrait qu'elles lui donnent des certitudes; et il est décontenancé, non tant par la discontinuité des structures archéologiques, que par les hésitations que manifestent les historiens et des archéologues lorsqu'il s'agit de les interpréter. Car malgré le fait qu'il a vu les sites qui comptaient parmi les mieux conservés de Grèce à cette époque, tout n'est pour lui qu'incertitude. C'est une constante chez L. Durrell qui évoque la difficulté de restituer la réalité avec d'aussi maigres témoins, en imaginant l'éventail des possibles qui s'offrirait à un archéologue minoen placé devant les vestiges éparpillés d'une Londres dévastée par

15 Par rapport aux paysages domestiqués, apprivoisés de l'Italie " finie comme un essai sur l'humanisme": Vénus et la mer, p. 226 ; Citrons acides, p. 106 : "En effet, le péristyle de l'abbaye formait un vibrant contraste avec ses orangers chargés de fruits et ses magnifiques berceaux de fleurs...".

16 Vénus et la mer, p. 171.

17 Les îles grecques, p. 142-143: "Seule l'absence de ruines donne à l'ensemble un aspect un peu fictif." "

18 Le grand suppositoire, p. 143.

19 F. Stark, Ionia. A Quest, Londres-New York, 1954, édition utilisée Tauris Parke Paperback, 2010, p. 20 : «I am one of those who prefer neglected ruins, places untouched even by the archaeologist, where one's thoughts can build their own places, and the past, draped and veiled in tis garment of earth, lies like the sleeping beauty undiscovered and undisturbed "; ou encore EAD., Baghdad Sketches. Journeys through Iraq, 1938, édition utilisée Marlboro Press, 1992, p. 159 : "For my own part, not being an archaeologist, I like ruins merely as places to sit about and think in... ". À ce propos, cf. R. EISNER, Travelers to an Antique Land. The History and Literature of Travel to Greece, Ann Arbor, 1991, p. 231-232.

20 Dans L'île de Prospéro, présence d'une chronologie en annexe ; dans Vénus et la Mer, p. 48, Platon est le marqueur chronologique de l'Antiquité ; dans Les îles grecques, p. 86, il propose un tableau chronologique de la civilisation crétoise ; cf. aussi L'esprit des lieux, p. 126 (lettre à Anne Ridler du 17 août 1952, à propos des jalons chronologiques de Rhodes). 
une explosion atomique ${ }^{21}$. À plusieurs reprises, il évoque les «balbutiements " ou les " bégayements " des érudits (angl. scholars, terme qui, sous sa plume, semble fort dépréciatif), des historiens et des archéologues ${ }^{22}$. Ce trait a déjà été souligné par Jean-Marc Riaume dans un récent article ${ }^{23}$.

Vénus et la mer (Reflections on a marine Venus) occupe une place à part dans l'œuvre de voyage envisagée sous l'angle de son rapport à l'histoire et à l'archéologie. Son sous-titre A Companion to the Landscape of Rhodes en dit long sur l'objectif didactique, voire scientifique de son auteur ; c'est le seul de ses ouvrages qui s'achève sur une bibliographie relative à l'histoire de Rhodes. D'autre part, le sujet même se prêtait mieux que n'importe quel autre à une réflexion sur l'archéologie, l'histoire et les restaurations. L'île avait été fouillée - et pour certains sites, reconstruite - par les Italiens (principalement Amedeo Maiuri et Luigi Morricone). Elle est donc pour lui le terrain de toutes sortes de spéculations. Sur les archéologues italiens, tout d'abord. Il leur reconnaît une rare qualité : l'honnêteté, qui les porte à traiter les vestiges grecs comme ils l'auraient fait de ruines romaines. Sur le goût des archéologues italiens en fait de restaurations, ensuite : on le sent plus que réservé sur la question de leurs anastyloses à Rhodes et à Lindos, puisqu'il évoque les [archéologues] "Italiens malavisés ${ }^{24}$ "; pour lui, les restaurations intempestives d'édifices gigantesques relèvent clairement d'une forme de folie ${ }^{25}$, voire

21 Les îles grecques, p. 85 : "Quelles seraient un jour les impressions d'un archéologue minoen fouillant un tas de boue sur l'emplacement de Londres dévasté par une explosion atomique ? Quand il aurait trouvé des objets aussi disparates qu'un ours en peluche, un père Noël, un Rembrandt (l'Angleterre était-elle donc pleine de singes, et à quelle époque ?), une croix de fer, une déclaration d'impôt sur le revenu, etc., qu'en conclurait-il ? Comment ferait-il pour trier tout cela et assigner une fonction à chaque objet? Les Anglais avaient-ils un ours pour totem ? Le père Noël était-il une sorte de Zeus ? La marge d'erreur possible est inquiétante et devrait nous mettre un peu en garde contre les "certitudes" dont parle T. S. Eliot. "

22 Voici par exemple, une belle "sortie " de L. Durrell à propos des archéologues dans L'esprit des lieux, p. 318 ("Delphes ») : "Le caractère spéculatif des assertions des érudits a de quoi vous fendre le cœur. C'est comme si rien n'était plus prouvable, comme si tout était devenu imprécis, provisoire. L'érudit se met à bégayer ; il peut difficilement nous dire quoi que ce soit sans employer les mots "probablement", "peut-être". Parfois, avec une grande audace, il se rapproche d'une phrase telle que "il paraît vraisemblable que..." ". Et ibid., p. 318 : « Mais l'oracle, la Pythie ? Encore une fois, les historiens se mettent à balbutier. »

23 J.-M. Riaume, "Lawrence Durrell et les îles de la Méditerranée ", Cycnos 7, mis en ligne le 9 juin 2008, URL : http://revel.unice.fr/cycnos/index.html?id=1159 (site consulté le 16 août 2012).

24 Vénus et la mer, p. 104.

25 Les îles grecques, p. 148 : «Des fouilles italiennes importantes menées entre 1916 et 1926, c'est-à-dire longtemps avant la folie de restaurer le château des Croisades, avaient révélé les fondations des temples de Zeus et d'Athéna Poliade...". 
de perversion ${ }^{26}$; jugement sévère, mais qui n'est pas très éloigné de celui qu'il défend dans ses Îles grecques ${ }^{27}$ sur les restaurations d'Arthur Evans à Cnossos, et qu'il partage par ailleurs avec une bonne part de l'intelligentsia de son époque ${ }^{28}$. Enfin, sur l'interprétation donnée par les archéologues et les restaurateurs des ruines à restaurer : là aussi, son jugement est sans appel puisqu'il évoque une reconstitution de Rhodes davantage animée par des préoccupations paysagistes que par la vraisemblance historique ${ }^{29}$.

\section{Lawrence Durrell et les archéologues}

Ce que L. Durrell reproche finalement aux "braves mastodontes " que sont les archéologues, c'est leur ignorance des réalités, qui ne les empêche cependant pas de creuser à tort et à travers ${ }^{30}$. Car, ainsi qu'on le découvre dans Vénus et la mer, les archéologues ne sont rien moins que des violeurs (angl. trespass), sans que ce viol perpétré à la pioche ${ }^{31}$ aboutisse à une plus grande compréhension du site, car l'archéologue rend souvent obscur ce qui ne l'est pas. Dans Le Carrousel sicilien - ouvrage tardif dans la production durrellienne - il observe que les archéologues conservent trop de choses, qui s'en vont

26 Correspondance, p. 297, à H. Miller, lettre du 22 juin 1945 : «Les Italiens, dit-on, sont des restaurateurs de grande valeur, mais je pense, moi, qu'ils sont plutôt des "arrangeurs" de paysage. Cette île minuscule n'est qu'une masse de petits coins artistement entourés de murs, décorés de jardins encombrés d'un fouillis de bougainvillées pourpres : tout cela est trop pensé." "

27 Les îles grecques, p. 142 : «Mais, bien sûr, si les techniques de remise au goût du jour adoptées par Evans à Cnossos nous font nous cabrer, la restauration de Rhodes doit faire l'objet de critiques du même genre. "

28 Par exemple R. Caillois, "Jeux d'ombres sur l'Hellade. Styles de vie du monde minoen ", Le voyage en Grèce 6 (1936), p. 14-20, particulièrement p. 19 : "Les aspects opposés et parents décrits tout à l'heure se superposent perceptiblement en ces lieux, dont la voyante crudité est encore rehaussée par le ciment des restaurations d'Evans. La complication et le mystère s'y marient avec l'ostentation et l'apparat. "

29 Les îles grecques, p. 142 : «La reconstitution fut admirablement exécutée, avec tout le bon goût des Italiens en matière d'architecture paysagiste et décorative. En fait, il n'est même pas à son avantage qu'elle ait l'air d'un décor de cinéma prêt pour un tournage. "

30 L'̂̀le de Prospéro, p. 81 (12 septembre 1937) : "Tels de braves mastodontes pétrifiés dans les forêts de leur propre attirail, les archéologues vont et viennent, avec leur Odyssée dans la poche et leur ignorance du grec moderne. Fouillant avec ardeur dans les tas de détritus de quelque antique bourgade, ils dressent, à partir de quelques tessons ou d'un vestige d'égouts le portrait maladif d'une civilisation."

31 Vénus et la mer, p. 171 : "Ils s'élèvent en une longue procession sur les sentiers crayeux de la ville jusqu'au mur de terre rouge que l'archéologue n'a pas violé, jusqu’à la colonne votive d'une poésie exagérée qui, on le devine sans peine, est l'œuvre des restaurateurs italiens. » 
finir dans des musées-cimetières ${ }^{32}$. De ce point de vue, il n'est pas éloigné d'H. Miller qui, en juin 1939, sur le bateau qui l'amène en Grèce, fait la connaissance d'un archéologue français, probablement membre de l'École française d'Athènes ; manifestement, le courant ne passe pas entre eux et, à l'escale de Naples, H. Miller, qui redoute une ennuyeuse visite de Pompéi en compagnie de l'archéologue, s'éclipse afin de visiter le site sans lui ${ }^{33}$.

Ainsi que les sites fouillés se répartissent, selon L. Durrell, en "sites parlants " (comme Cnossos, Mycènes, Delphes, Epidaure, Rhodes) et "sites confus » (par exemple, Argos, Eleusis), les archéologues se groupent-ils en deux catégories : d'une part les "bredouilleurs ", les archéologues qui ne s'expriment que moyennant moult précautions oratoires ("vraisemblablement ", " peut-être »... : autrement dit, le style des rapports archéologiques et d'une certaine littérature archéologique qui tente de s'approprier les méthodes et les moyens d'expression des sciences dures). Ces archéologues-là sont des érudits farcis de lectures savantes mais qui ne tranchent jamais et peinent à donner au lecteur un résumé simple et solide. Et d'autre part, il y a les "éclaireurs ", ceux qui sont attendus sur des sites comme Argos, pour en être les véritables interprètes ${ }^{34}$. À cette catégorie appartiennent quelques rescapés, qui ont presque tous comme point commun de compter parmi les relations personnelles de l'écrivain : probablement ces archéologues-là ont-ils eu l'occasion de lui expliquer de vive voix leurs objectifs, leurs méthodes et les résultats auxquels ils avaient abouti. De fait, ce sont aussi des savants qui ont classé, rangé, expliqué, restitué, ou reconstruit, bref, qui ont mis en ordre le réel. Les "deux étoiles permanentes au firmament de l'archéologie grecque ${ }^{35}$ " sont, pour L. Durrell, Heinrich Schliemann et surtout Arthur Evans (qu'il connaît personnelle-

32 Le Carrousel sicilien, p. 122 : «Il y a là [au musée de Syracuse] un extraordinaire ramassis de fragments de poterie et de pierre de toutes sortes, pour la plupart sans aucun intérêt esthétique et simplement conservés comme reflets d'une époque ou d'une tendance. Il existe de nos jours d'immenses cimetières de ce genre de vestiges..." ".

33 H. Miller, Le colosse de Maroussi, trad. française G. Belmont, Paris, 1972, p. 9. « Il y avait un archéologue français qui retournait en Grèce. À table, il était assis en face de moi. Il aurait pu me dire des tas de choses sur le pays. Je ne lui en laissai jamais la chance. Il me déplut au premier coup d'œil. [...] À Naples, nous descendîmes à terre ensemble pour faire un bon repas et visiter Pompéi, dont il [l'Italien d'Argentine pour qui il se prend d'amitié] n'avait pas même entendu parler. Il faisait une chaleur écrasante ; n'empêche que la balade à Pompéi me plut énormément. Si j’y étais allé avec l'archéologue, je me serais affreusement ennuyé." "

34 L'esprit des lieux, p. 65 : "Dès notre retour, nous nous plongeâmes dans l'histoire et l'archéologie de la plaine d'Argos. Tout cela se trouve dans un si triste état de confusion! Ce n'est qu'un tissu de conjectures et d'hypothèses fondées sur des grains de riz et sur des traces de crottin de cheval. Ce n'est pas cela. Ces lieux attendent toujours leurs véritables interprètes ; même ce sinistre Eleusis boueux avec ses affreuses pompes à essence. "

Les îles grecques, p. 84. 
ment ${ }^{36}$ ), qui a le mérite de rendre les vestiges parlants ${ }^{37}$. On trouve également le nom de celui qui fut directeur de la British School of Archaeoloy at Athens de 1906 à 1914, Richard MacGillivray Dawkins (fouilleur, mais aussi spécialiste du grec byzantin) ${ }^{38}$, et celui de Peter Megaw ${ }^{39}$, directeur du département des Antiquités de Chypre de 1936 à 1960, qui est remercié par L. Durrell dans la préface des Citrons acides. En poste au Caire, il se lie avec l'égyptologue américain George Andrew Reisner, qui lui fait la visite de la pyramide de la reine Hetepheres $\mathrm{I}^{40}$. Enfin, il y a l'archéologue écossais de Rhodes qui apparaît sous le pseudonyme de "Sand " dans Vénus et la mer. C'est le Major Theodore Burton Brown ${ }^{41}$, dont la thèse Early Mediterranean Migrations ${ }^{42}$ connaîtra un certain retentissement. De dix ans l'aîné de l'écrivain, Th. Burton Brown est décrit par L. Durrell comme de bon caractère mais " têtu " et taciturne ${ }^{43}$; au cours de ses deux années rhodiennes, l'archéologue compte parmi ses proches. À Rhodes, Th. Burton Brown a fait, ainsi qu'on le déduit des rapports annuels d'activités de la British School of Archaeoloy at Athens ${ }^{44}$, un travail de fouille dans les zones sinistrées par les bombardements mais surtout, de présentation des objets du musée qui, pendant la

36 Evans : entre autres, Correspondance, p. 71 (lettre de fin décembre? 1936) : «Si Evans était là, ce serait facile de lui rendre visite... ». Probablement se connaissent-ils parce qu'Evans fait partie des illustres expatriés britanniques de Grèce et que les deux hommes avaient eu l'occasion de se rencontrer à Athènes.

37 Les îles grecques, p. 84-85 : "C'était plutôt un gros travailleur qu'un imaginatif, encore qu'il eût écrit dans sa jeunesse un excellent mémoire de voyage sur la Yougoslavie. Avec le recul du temps, on a l'impression que tout était à sa disposition et n'attendait que lui, toute une civilisation qui allait repousser plus loin la sombre frontière de la préhistoire ». Ibid., p. 87 : «La question de savoir ce que vaut la restauration d'Evans ne peut manquer de se poser ; personnellement, je la trouve sans charme et de mauvais goût. Mais il faut tenir compte du fait qu'Evans voulait illustrer les rapports des monuments entre eux et que ce but est atteint."

38 Correspondance, p. 71 (lettre de fin décembre? 1936), où il dit à H. Miller qu'il a rencontré R. M. Dawkins chez Evans à Cnossos.

39 Arthur Herbert Stanley Megaw (connu sous le nom de Peter Megaw), né en 1910, mort en 2006 : P. Flourentzos, "In memory of A. H. S. Megaw », Report of the Department of Antiquities, Cyprus (2006), p. i.

40 Cf. Th. Stephanides, Autumn Gleanings. Corfu Memoirs \& Poems, Corfou, 2011, p. 81-82.

41 J. Leatham, "Durrell on Rhodes", dans A. Lillios, Lawrence Durrell and the Greek World, Susquehanna, 2004, p. 145-152, et particulièrement p. 149.

42 Publiée à Manchester en 1959. Après les années rhodiennes, Th. Burton-Brown (né en 1902) semble avoir tourné la page de l'archéologie grecque, puisqu'il fera une carrière sur des chantiers proche-orientaux et orientaux (en Iran, Iraq et Géorgie).

43 Vénus et la mer, p. 53 ; ibid., p. 248.

44 Cf. T. W. French, " Notes. Archaeology in the Dodecanese 1939-1946 ", Journal of Hellenic Studies 65 (1945), p. 101-102 (dans la cité fortifiée de Rhodes et notamment près de la porte de Saint-Athanasios, où fut découvert le règlement relatif au sanctuaire d'Asclépios, dont il donne une photographie) : ibid., p. 101 et 102, fig. 1. 
Seconde Guerre mondiale, avaient été mis à l'abri dans les réserves. Il mit sur pied une exposition des œuvres ainsi remontées des sous-sols ${ }^{45}$ et notamment la plus attachante des statues - du moins du point de vue du romancier - celle de la Vénus marine ${ }^{46}$ : ici encore, un travail de remise en ordre et de clarification des artefacts qui devait rendre les réalités archéologiques plus parlantes.

\section{Durrell archéologue}

Son attitude personnelle vis-à-vis de la profession d'archéologue reflète aussi toute l'ambiguïté de ses rapports à l'archéologie. En effet, malgré la piètre opinion qu'il a du vulgum pecus archéologique, L. Durrell n’hésite pas à jouer à l'archéologue. À Chypre, il se fait passer pour un archéologue - allemand - lorsqu'il constate que, malgré sa maîtrise du grec, il ne s'attire, en tant qu'Anglais, que de l'antipathie de la part des clients de tavernes locales ${ }^{47}$. Cette fonction neutre, nimbée d'autorité, encore réservée aux étrangers dans la Chypre de la fin des années 50, lui sert de tenue de camouflage pour s'approcher masqué d'un interlocuteur chypriote soupçonneux. Mais c'est surtout l'idée de restituer le passé grec et romain qui le conduit à en rechercher les traces matérielles. Ainsi, à plusieurs reprises, évoque-t-il ses ramassages archéologiques : il donne au musée de Rhodes des récipients trouvés sur le site de Camiros $^{48}$; dans le port de Rhodes, il recherche intensivement des restes du colosse ${ }^{49}$. À Corfou, il plonge afin de remonter des vestiges de ce qu'il croit être l'ancienne villa d'été de Tibère à Cassopi ${ }^{50}$ et à Analypsis, il tente de repérer les ruines d'un temple de Poséidon qui aurait été vu par les officiers de la marine britannique à la fin du XIX ${ }^{e}$ siècle ${ }^{51}$. Enfin, les rudiments

45 J. CooK, "Archaeology in Greece, 1945-1947 ", Journal of Hellenic Studies 67 (1947), p. 117 : "The greater part of the museum collections is still in store, but an exhibition of sculptures and select vases was arranged in the Rhodes Museum by Major T. Burton Brown. "

46 Vénus et la mer, p. 42-43.

47 Citrons acides, p. 253 : "J'étais environné de regards sombres et hostiles, où passait une fugitive lueur lorsque je disais quelques mots de grec. Dans le troisième café, je dis que j'étais un archéologue allemand et aussitôt, l'atmosphère parut se détendre. "

48 Les îles grecques, p. 166-167 ; découverte de poteries à Camiros, Vénus et la mer, p. 147.

49 Vénus et la mer, p. 130 : "Quant à moi, j’ai passé des journées entières à en discuter ; des après-midi entiers à plonger dans les eaux du port à la recherche de quelque fragment de la grande statue (m'appuyant en cela sur la folle théorie de Gideon selon laquelle elle était située sur la jetée est)...".

50 Correspondance Durrell-Miller 1935-1980, p. 70-71 (fin décembre? 1936) ; L'esprit des lieux, p. 54 (lettre à Alan G. Thomas, 1937) ; L'̂̀le de Prospéro, p. 89-90 (25 septembre 1937).

51 Lî̀le de Prospéro, p. 113 (16 novembre 1937) ; J.-M. Riaume, "Lawrence Durrell et les îles de la Méditerranée " (supra, n. 23), n. 33. 
qu'il avait du grec ancien - étudié pendant deux ans en Angleterre ${ }^{52}$ - lui permettent de se frotter à l'épigraphie, lorsqu'avec Th. Burton Brown, il déchiffre et interprète une inscription grecque récemment découverte ${ }^{53}$. Les inscriptions grecques antiques retiennent d'ailleurs souvent son attention et, à plus d'une reprise, il en résume le contenu pour le lecteur, attirant son attention sur tel ou tel mot, caractéristique d'un formulaire antique ${ }^{54}$.

Sa correspondance privée témoigne d'ailleurs de son réel souci d'exhaustivité dans l'information qu'il donne (notamment dans Vénus et la mer) : il veut faire comprendre à son lecteur le sens des vestiges archéologiques. Ainsi, dans une lettre à Anne Ridler, - chargée de publier son livre sur Rhodes -, il s'étonne qu'elle ait supprimé ce qui touchait au second siège de Rhodes. "Je ne peux m'empêcher de penser que si l'on devait passer une semaine à Rhodes, en utilisant ce livre comme guide, on se dirait : "Bon sang! Pas un mot sur la ville médiévale et elle est là sous nos yeux !" Et la carte que je veux reproduire et qui indique si minutieusement chaque site... Imaginez un livre sur Oxford qui ignorerait les monuments actuels et ne mentionnerait que les vestiges anglo-saxons dont la plupart ne sont pas visibles à l'œil nu ${ }^{55}$ ? " Ici encore, L. Durrell fait pour le lecteur non averti mais intéressé ce qu'il voudrait que les historiens fissent pour lui.

Tout ceci fait de L. Durrell une source littéraire non négligeable en matière d'histoire ancienne et d'archéologie, à décrypter en comparant les récits, la correspondance et les interviews.

\section{Ses sources d'information}

Sa bibliographie est anglophone et principalement anglaise, plus rarement française. Si les ouvrages cités en bibliographie de Vénus et la mer sont parfois un peu anciens (E. Dodwell, A classical and topographical tour of Greece, 1819, ou E. Billiotti et l'abbé Cottret, L'̂̀le de Rhodes, 1881, et les ouvrages de C. Torr, Rhodes in Ancient Times, 1885 ; Rhodes under the Byzantines, 1886 ; Rhodes in Modern Times, 1887) ou généraux (on pense au livre de C. E. Robinson, Everyday life in Ancient Greece, Oxford,

52 C. Alexandre-Garner, Lawrence Durrell, p. 54 (entretien avec C. Alexandre-Garner ; supra, n. 3).

53 Vénus et la mer, p. 128 : «Une inscription récemment mise au jour par Sand interdisait formellement aux Rhodiens d'ajouter une autre statue au temple d'Esculape. »

54 Vénus et la mer, p. 171 (inscriptions de Camiros avec le nom des pères de la cité, des prêtres et des suppliants) ; ibid., p. 172 (toujours à Camiros, les inscriptions funéraires et leur vocabulaire) ; ibid., p. 185 (inscription reprenant l'ode de Pindare sur Diagoras de Ialysos) ; Les îles grecques, p. 74 (consacre un long développement à l'inscription de Gortyne en Crète).

55 L'esprit des lieux, p. 126 (lettre du 17 août 1952). 
1933, qu'il souhaite qu'on lui envoie alors qu'il est en poste à Belgrade ${ }^{56}$ ), il réactualise ses données avec les ouvrages plus récents qu'il consulte dans la bibliothèque de Th. Burton-Brown ${ }^{57}$ (ou plutôt la bibliothèque du FERI, l'Institut d'histoire orientale de Rhodes, installée dans une annexe du musée ${ }^{58}$ ), ou dans la salle à rotonde du British Museum qu'il fréquente lors de ses retours à Londres ${ }^{59}$. Probablement rafraîchit-il aussi ponctuellement ses informations avec les Archaeological Reports publiés dans le Journal of Hellenic Studies, mais il ne les cite pas explicitement. La littérature archéologique " pointue " - mais relativement indigeste pour le profane - n'était en effet étrangère ni à L. Durrell, ni à H. Miller : en visite à Mycènes, ce dernier s'était installé à une terrasse avec " un bon gros livre d'archéologie de l'école anglaise en guise de hors-d'œuvre ${ }^{60}{ }^{\prime}$.

On retiendra quelques grands noms, nommément cités dans Les îles grecques et dans sa Correspondance : à nouveau, le nom le plus souvent mentionné est celui d'Arthur Evans. Cité à la fois par L. Durrell et par H. Miller, The Palace of Minos at Knossos semble être leur livre de chevet ${ }^{61}$, ce qui en dit long sur l'effet qu'avaient produit les découvertes d'A. Evans sur le monde cultivé de l'époque. Dans Les îles grecques, on trouve aussi les noms de l'archéologue Jacquetta Hawkes ${ }^{62}$ et de Robert Graves ${ }^{63}$, auteur de nombreux ouvrages académiques sur la littérature, et notamment d'un livre consacré aux mythes grecs. Ces deux auteurs présentent cependant la particularité d'être tous deux liés au monde des lettres, puisque la première est l'épouse du dramaturge John Boynton Priestley et que le second était poète de renom. Le nom de Moses Finley apparaît dans Les îles grecques ${ }^{64}$. Enfin, l'historien Ernle Bradford est cité dans la préface aux Îles grecques (pour son Companion Guide to the Greek Islands, 1963) et c'est encore à l'une de ses publications que L. Durrell fait allusion, lorsqu'il évoque le plan au sol de la cité antique de Rhodes, révélé par photographie aérienne ${ }^{65}$. L. Durrell s’informe donc dans des sources historiques de bonne qualité, et complète sa documentation avec des lectures archéologiques plus pointues, mais retient aussi les informations qui

56 L'esprit des lieux, p. 124 (lettre à Alan Thomas, 1952).

57 Vénus et la mer, p. 51.

58 Rapport N. Vlachos du 30 septembre 1946 (dans L. Divani et Ph. Constantopoulou, The Dodecanese. The Long Road to Union with Greece, Athènes, 1997, p. 201).

59 C. Alexandre-Garner, Lawrence Durrell, p. 70 (supra, n. 3).

60 H. Miller, Premiers regards sur la Grèce, Santa Barbara, 1973, trad. française C. CHICHEREAU Paris, 1999, p. 33.

61 Les îles grecques, p. 74.

62 Cités dans Les îles grecques, p. 76. Il s'agit probablement de l'ouvrage de J. HawKES intitulé Dawn of the Gods, Londres, 1968.

63 The Greek Myths, Londres, 1965.

64 Les îles grecques, p. 115.

65 Les îles grecques, p. 148 ; E. BRADFORD, "Fieldwork on aerial discoveries in Attica and Rhodes. Part I. The town plan of Classical Rhodes ", The Antiquaries Journal 36 (1956), p. 57-69. 
lui sont données de vive voix, qu'elles viennent d'archéologues confirmés ou de savants locaux, comme l'écrivain Gostan Zarian, source d'informations majeure dans L'île de Prospéro ${ }^{66}$, ou le docteur Theodore Stephanides, homme "d'une érudition incroyable pour tout ce qui concerne l'île ${ }^{67}{ }^{\prime}$.

\section{Durrell, une source historique?}

Si L. Durrell apparaît donc comme un auteur soucieux de diffuser des informations justes, il est des cas où il devient à son tour une source d'information. Il reste à déterminer si cette source est fiable ou non, et dans quelles circonstances. Le premier cas que nous envisagerons est celui de la Rhodes d'après-guerre dans laquelle L. Durrell, envoyé comme officier chargé de surveiller l'information, se lie d'amitié avec le directeur des antiquités du Dodécanèse, le Major Burton-Brown cité plus haut. Tous deux sont mandatés par l'Administration militaire britannique et resteront en poste d'avril 1945 à février 1947. Contrairement à son habitude qui est de nommer les gens tantôt par leur nom (Egon Huber, le docteur Theodore Stephanides, Mills, Zarian...), tantôt par l'initiale de leur prénom (E.[ve], N.[ancy]...), ou encore d'abréger leur nom (Hoyle/ Hoyl $[$ and $\left.]{ }^{68} \ldots\right)$, L. Durrell utilise ici un pseudonyme sans lien direct avec le nom du personnage ${ }^{69}$. L. Durrell paraît donc nimber sciemment de mystère ce directeur des antiquités : ceci peut s'expliquer par la nature militaire de sa fonction, dont, par ailleurs, il ne dit mot.

Rappelons tout d'abord le climat tendu dans lequel L. Durrell et Th. BurtonBrown prennent leurs fonctions. L'île désertée de l'après-guerre est désormais en proie

66 Lîle de Prospéro, p. 16-17 : description de Zarian et de son cabinet de travail : « Il a réussi à transformer le dernier étage de l'hôtel Saint-Georges en cabinet de travail : un fouillis de manuscrits et de peintures. C'est là, devant les fortifications trapues du Fort Oriental, qu'il compile ses articles littéraires pour divers journaux arméniens du nouveau monde, en s'arrêtant de temps à autre pour vider un verre de vin »; p. 111 (entre autres) " "Pour Napoléon", dit Zarian qui se délecte des grands noms, "Corfou était la clé de voûte d'un Empire d'Orient"...".

67 L'île de Prospéro, p. 18.

68 J. Leatham, "Durrell on Rhodes " (supra, n. 41), p. 145-152, 149. Il s'agirait du consulgénéral de Grande-Bretagne en Grèce, Harold Allan Dilke Hoyland, nommé à ce poste le 14 juin 1945 (Supplement to the London Gazette, 14 juin 1945, p. 2954).

69 Ce nom pourrait être inspiré du nom du juge Emil Sandström (1886-1962), président de la Greek Relief Commission en 1944 : il fit de nombreux déplacements en Grèce et dans les îles et devint, en 1947, membre de l'United Nations Special Committee on Palestine (UNSCOP), avant d'être nommé, en 1950, à la tête de la Croix Rouge de Suède. Ses compétences en fait de justice et de médiation en firent un homme aimé de tous et ce patronyme devait être relativement familier à l'écrivain. 
à la guerre civile ${ }^{70}$. De tout cela, L. Durrell ne fait pas état - en tout cas dans Vénus et la mer, car dans Les îles grecques, il évoque la famine qui toucha l'île à la fin de la guerre et le chaos qui lui succéda ${ }^{71}$. Du point de vue de l'archéologie, l'ambiance n'est pas non plus des meilleures : les Grecs soupçonnent les Italiens d'avoir volé des antiquités pendant les années d'occupation ${ }^{72}$; par ailleurs, la mise sous la tutelle de l'Administration militaire britannique du Dodécanèse et de son patrimoine devait également susciter des rancours. Ici encore, L. Durrell ne dit rien de ces difficultés, mais on devine que le caractère " têtu » de l'archéologue a peut-être pu poser quelques problèmes, entre autres dans ses relations avec les Grecs chargés de préparer la transition. Nous disposons à ce propos de deux sources de nature diplomatique : le rapport de N. Vlachos ${ }^{73}$, rédigé en septembre 1946, à destination des ministères grecs de l'Éducation nationale et des Affaires étrangères, et le rapport du gouverneur anglais A. S. Parker ${ }^{74}$, qui dresse un bilan de ces deux années de tutelle britannique. Si ce dernier montre que l'officier britannique (qu'il ne nomme pas) responsable de l'archéologie a multiplié les activités (fouille, recensement des inscriptions dans les îles, dont plusieurs inconnues, mise sur pied d'une exposition consacrée à l'histoire de Rhodes de 1500 avant n. è. à l'époque des Chevaliers, ouverture du musée au public, initiatives touchant à la gratuité des musées, reclassement de livres et d'archives photographiques, publication de guides...), il en va tout autrement du rapport du professeur N. Vlachos. Ne citant jamais nommément Th. Burton Brown - qui n'apparaît que sous l'expression consacrée « The British archaeologist and Director of the museum »-, il semble minimiser son activité en tant que directeur. Si l'on en croit ce rapport, le travail du directeur du musée se résumait à établir des estimations financières pour des restaurations; le rapport sous entend aussi

70 On consultera à ce propos l'article de Murielle PHiLippe, "Reflections on a Marine Venus (1953) : le détour par Rhodes dans la trilogie des îles de Lawrence Durrell », E-rea [En ligne], 3.1 | 2005, mis en ligne le 15 juin 2005, URL : http://erea.revues.org/535 (site consulté le 16 août 2012).

71 Les îles grecques, p. 145 "Il n'était pas ordinaire de voir les animaux familiers attachés à la poignée de la porte d'entrée, de crainte qu'ils ne s'échappent et ne soient consommés par les soldats qui pillaient à droite et à gauche ; chiens, chats ou cobayes, tout ce qui leur tombait sous la main finissait dans la marmite ". Ibid. : "Rhodes se réveilla donc comme une Belle au bois dormant, mais il fallut plus d'un baiser poilu de la nouvelle administration militaire pour la remettre sur pied. C'était un chaos inimaginable.»

72 Rapport Vlachos du 30 septembre 1946 (repris dans L. Divani et Ph. Constantopoulou, The Dodecanese. The long road to union with Greece, Athènes, 1997, p. 201), on lit l'histoire du marin Mikhaill Petridis qui, en 1943, alors qu'il servait sur le "Doukessa », navire réquisitionné par les Italiens, il convoya des antiquités vers l'Italie.

73 Rapportdu30 septembre 1946; repris dansl'ouvrage deL. Divani et Ph. Constantopoulou, The Dodecanese, p. 200.

74 Rapport de A.S. Parker, "Report of the Governor A. S. Parker on the Acts of the British Military Administration 1/1/1946-31/12/1946), chap. XXI, Antiquities (dans L. DivanI et Ph. Constantopoulou, The Dodecanese, p. 281). 
qu'interrogé avec insistance sur la tenue de catalogues et d'inventaires des collections, le directeur aurait cherché à tergiverser... et n'aurait pas rempli sa mission de conservation des collections ${ }^{75}$. Bref, ce rapport peu louangeur laisse entendre que l'activité de Th. Burton-Brown en tant que directeur n'aurait été qu'une parenthèse relativement stérile - entre les "pillages italiens » et la reprise en main, ardemment souhaitée, du service, en avril 1947, par l'Administration militaire grecque.

On ajoutera que même dans l'introduction de son ouvrage sur l'histoire de Rhodes, G. Konstantinopoulos élude la question de l'histoire du musée au cours des deux années où Rhodes fut sous administration militaire britannique ${ }^{76}$. Enfin, outre le rapport officiel du British Committee on the Preservation and Restitution of Works of Arts, Archives and other Material in Enemy Hands ${ }^{77}$, une compilation de rapports archéologiques où n'apparaît pas le nom de l'archéologue britannique de Rhodes, le premier rapport archéologique un peu disert sur la question est celui de T. W. French ${ }^{78}$, qui souligne le travail de conservation des vestiges accompli pendant ces deux années. Mais finalement, le seul document officiel à mentionner en toutes lettres le nom et l'activité de Th. Burton-Brown est le rapport annuel d'activités de la British School of Archaeology des années 1945-1947, signé de J. Cook ${ }^{79}$.

C'est ici que l'œuvre de L. Durrell apporte des indications très intéressantes touchant à l'histoire de l'archéologie et de la muséologie à Rhodes qui recoupent le rapport du gouverneur A. S. Parker - puisque L. Durrell est le seul à donner son titre de "directeur des Antiquités de Rhodes ", un poste à responsabilités, donc -, mais aussi à conserver, en filigrane, égrenées au fil des pages de Vénus et la mer, des traces du travail mené par Th. Burton-Brown, et notamment de ses activités de conservateur, de fouilleur et d'épigraphiste. L'allusion au déchiffrement de l'inscription trouvée par l'archéologue à la porte d'Aghios Athanasios dans la vieille ville de Rhodes donne un exemple intéressant de ce qui se produisit. Découverte par Th. Burton-Brown, déchiffrée par lui (et L. Durrell...) ainsi que ce dernier le relate dans Vénus et la mer ${ }^{80}$, l'inscription est, la même année, mentionnée dans le rapport annuel du directeur de l'École

75 Ibid. (L. Divani et Ph. Constantopoulou, The Dodecanese, p. 200-201) : "The British Archaeologist and Director of the Museum, repeatedly asked by me whether there catalogues of the contents of the archaeological finds and items of the Museum of Rhodes and Cos, sought to prevaricate. He merely assured me that the archaeological finds and the items of folk art have been carefully guarded since the beginning of the war and that there is no cause of concern."

76 G. Konstantinopoulou, Arkaia Rhodos, Athènes, 1986, p. 13.

77 Works of art in Greece. The Greek Islands and the Dodecanese. Losses and Survivals in the War, Londres, 1946, p. 55-63 (pages relatives au Dodécanèse).

78 T. W. French, "Notes. Archaeology in the Dodecanese 1939-1946 " (supra, n. 44), p. 101-104 : " numerous finds were made but work was concentrated on preservation, and excavation was only undertaken when it was clearly essential to do so. »

79 J. Соок, "Archaeology in Greece, 1945-1947» (supra, n. 45), p. 117.

80 Vénus et la mer, p. 128. 
britannique (il en donne une photo mais ne mentionne ni le découvreur, ni la première lecture qui en a été faite) ${ }^{81}$; l'année suivante, L. Robert en signale l'importance du point de vue de l'histoire de la pratique religieuse grecque - c'est un règlement qui interdit de placer des statues à un endroit du sanctuaire d'Asclépios à Rhodes, où elles gêneraient les promenades -, la restitue et la commente ${ }^{82}$. Enfin, G. Pugliese-Caratelli en assure la publication scientifique, l'insérant dans un ensemble de cent neuf autres inscriptions rhodiennes mises au jour avant-guerre ${ }^{83}$. La plus intéressante découverte épigraphique faite au cours de ces années a donc ainsi progressivement échappé à son découvreur, rejoignant discrètement le lot d'inscriptions mises au jour lors des fouilles italiennes. Au-delà du récit plaisant d'un séjour rhodien et des anecdotes relatives à telle ou telle promenade archéologique, le texte de L. Durrell se révèle donc être une source d'informations de première main touchant à un moment douloureux de l'histoire et de l'archéologie du Dodécanèse, où le contrôle sur le patrimoine se révèle un enjeu majeur pour la nouvelle administration militaire grecque.

Par contre, l'ironie du sort ou la notoriété de L. Durrell ont voulu qu'il soit devenu à son tour une source littéraire sur l'archéologie - en tout cas pour les touristes et certains utilisateurs du web -, mais une source hélas fausse. Sur le site de Cass(i)opi, à la pointe septentrionale de Corfou - non loin de Calami, l'endroit où les Durrell habitaient pendant leurs années corcyréennes -, il y aurait, selon L. Durrell, les restes d'une des villas d'été de Tibère. C'est une idée qu'il développe très tôt, dès 1939, dans L'île de Prospéro, qu'il évoque à plusieurs reprises dans ses Îles grecques; en 1983, encore, en voyage à Corfou avec la comédienne Laure Casteil, il lui montre les « ruines du palais de Tibère ${ }^{84}$. " Il fait ailleurs des considérations d'ordre topographique, mettant en parallèle le choix de Cassopi avec celui de Rhodes ${ }^{85}$, où Tibère avait séjourné.

L'origine de cette légende n'est pas claire. On dénombre au moins trois toponymes Cass(i)opi dans cette région : un promontoire et un port sur l'île de Corcyre, et un port sur la côte épirote, près de Buthrint ${ }^{86}$. Quant aux ruines de la Cassopi corcy-

81 T. W. French, "Notes. Archaeology in the Dodecanese 1939-1946 " (supra, n. 44), p. 102 , fig. 1.

82 J. et L. Robert, "Bulletin épigraphique », Revue des Études Grecques 61 (1948), p. 183, 172.

83 G. Pugliese-Carratelli, " Nuovo supplemento epigraphico Rodio », Annali della Scuola Archeologica di Atene 33-34 n. s., 17-18 (1955-1956), p. 157-181, et particulièrement p. 157, $\mathrm{n}^{\circ} 1$; voir plus récemment Supplementum Epigraphicum Graecum 55 (2005), $\mathrm{n}^{\circ} 907$ avec la bibliographie.

84 Interview accordée par Laure Casteil au Midi libre, article du 26 février 2012 mis en ligne http://sommieres.blogs.midilibre.com/archive/2012/02/26/les-cent-ans-de-lawrence-durrell.html (site consulté le 23 septembre 2012).

85 Les îles grecques, p. 31 et 152.

86 L. BÜrChnER, s.v. «Kassiope », RE, X 2, 1919, col. 2314-2315; en ce qui concerne le port corfiote dont parle L. Durrell, voir aussi D. Strauch, s.v. "Kassiope ", Der Neue Pauly, 6 (1999), p. 322. 
réenne, elles sont effectivement d'époque romaine ${ }^{87}$ et sont celles du temple de Zeus Cassios (temple connu des sources antiques, et mentionné par L. Durrell ${ }^{88}$ ). Aucune source antique n'associe Corcyre - et particulièrement Cassopi - à Tibère ; par contre, Suétone - entre autres - rapporte que Néron a chanté devant l'autel de Zeus Cassios $^{89}$. Identifier un auteur plus tardif (par exemple des ouvrages des XVIII ${ }^{\mathrm{e}}$ et $\mathrm{XIX}^{\mathrm{e}}$ siècles) comme origine à cette légende n'a pas non plus été possible. Nous en déduisons que les informations de L. Durrell viennent probablement d'une légende locale, voire d'informations peut-être transmises par Gostan Zarian, qui s'était installé à Corfou, et avec lequel il était lié pendant ses années corfiotes ${ }^{90}$. Néanmoins, à l'heure actuelle, Cassopi est associée - sur les sites web - à une villa de Tibère et on assiste, depuis la publication des écrits de L. Durrell et à cause de la notoriété dont il jouit en matière de " choses grecques ", à la création d'une fausse archéologie et d'une fausse histoire.

Par l'intérêt qu'il porte à l'archéologie, L. Durrell est un témoin privilégié de l'histoire et de l'histoire de l'archéologie telles qu'elles se firent dans l'est de la Méditerranée. Partagé entre son goût des paysages « sauvages " de Grèce, celui des ruines inviolées qui gardent leur secret et ses relations tissées avec quelques archéologues de grand format, il éprouve une soif ardente de comprendre (les traces du passé, l'histoire) et de faire comprendre : probablement, est-il même en cela un vrai romantique, ainsi qu'il se définit à M. Alyn : "Un romantique est éternellement en conflit avec lui-même ${ }^{91}$. " Aussi faut-il considérer comme une marque de reconnaissance le fait que ce soit à lui que les historiens Andrew Robert et Mary Burn aient confié la préface de leur ouvrage, The Living Past of Greece. A time-traveller's tour of historic and prehistoric places, publié à Londres en 1980. Ainsi, de simple observateur de l'archéologie L. Durrell est-il devenu un véritable expert en matière de ruines grecques.

Isabelle TASSIGNON

Université de Namur

Département d'Histoire de l'Art et d'Archéologie

61, rue de Bruxelles

B-5000 Namur (Belgique)

isabelle.tassignon@fundp.ac.be

87 Les vestiges les plus anciens semblent remonter à l'époque romaine : K. KostoglouDesPinI, "Anaskaphi is Kassiopin Kerkiras ", Archaiologika Analekta ex Athinon 4 (1971), p. 202-206 ; "Chronique archéologique ", Bulletin de Correspondance Hellénique 100 (1976), p. 621 ; outre le temple de Zeus Cassios, on a mis au jour les vestiges d'un autre bâtiment important daté des $\mathrm{III}^{\mathrm{e}}-\mathrm{IV}^{\mathrm{e}}$ s. : "Chronique archéologique ", Bulletin de Correspondance Hellénique 117 (1993), p. 808-809.

88 L'̂̀le de Prospéro, p. 97 (7 octobre 1937).

89 Tacite, Annales, XVI, 48 ; Suétone, Vies, Néron, XXII.

90 Th. Stephanides, Autumn Gleanings (supra, n. 40), p. 35, n. 28.

91 Le grand suppositoire (supra, n. 1), p. 107. 